\title{
Existence of multiple positive solutions for third-order $p$-Laplacian functional dynamic equations on time scales
}

\section{Wen Guan*}

\section{"Correspondence:}

mathguanw@163.com

Department of Applied

Mathematics, Lanzhou University of

Technology, Lanzhou, Gansu

730050, People's Republic of China

\begin{abstract}
In this paper, we consider a class of boundary value problems for third-order $p$-Laplacian functional dynamic equations on time scales, some existence criteria of at least three positive solutions are established. The main tool used in this paper is the fixed point theorem due to Avery and Peterson (Comput. Math. Appl. 42:313-322, 2001).

MSC: 39K10; 34B15

Keywords: time scale; $p$-Laplacian functional dynamic equations; boundary value problem; fixed point
\end{abstract}

\section{Introduction}

Recently, some authors have paid much attention to the existence of positive solutions for functional dynamic equations on time scales [1-8], especially for the $p$-Laplacian functional dynamic equations on time scales [1,3-8]. For convenience, throughout this paper we denote by $\Phi_{p}(s)$ the $p$-Laplacian operator, i.e., $\Phi_{p}(s)=|s|^{p-2} s, p>1,\left(\Phi_{p}\right)^{-1}=\Phi_{q}$, $\frac{1}{p}+\frac{1}{q}=1$.

In [2], Kaufmann and Raffoul considered a nonlinear functional dynamic equation on time scales and obtained sufficient conditions for the existence of positive solutions. In [5], by using a double fixed point theorem due to Avery et al. [9], Song and Gao considered the existence of at least twin positive solutions to the following $p$-Laplacian functional dynamic equations on time scales:

$$
\left\{\begin{array}{l}
{\left[\Phi_{p}\left(u^{\Delta \nabla}(t)\right)\right]^{\nabla}+a(t) f(u(t), u(\mu(t)))=0, \quad t \in(0, T)_{\mathrm{T}},} \\
u(t)=\varphi(t), \quad t \in[-r, 0]_{\mathrm{T}}, \\
u^{\Delta}(0)=u^{\Delta \nabla}(T)=0, \quad u(T)+B_{0}\left(u^{\Delta}(\eta)\right)=0,
\end{array}\right.
$$

where $\eta \in(0, \rho(T))_{\mathrm{T}},-r, 0, T \in \mathbf{T}$.

In [8], Wang and Guan considered the existence of positive solutions to problem (1.1) by applying the well-known Leggett-Williams fixed point theorem [10].

Motivated by $[2,5]$ and $[8]$, we shall show that problem (1.1) has at least three positive solutions by means of the fixed point theorem due to Avery and Peterson [11].

@2014 Guan; licensee Springer. This is an Open Access article distributed under the terms of the Creative Commons Attribution License (http://creativecommons.org/licenses/by/2.0), which permits unrestricted use, distribution, and reproduction in any medium, provided the original work is properly cited. 
In this article, we always assume that:

$\left(\mathrm{C}_{1}\right) f:[0,+\infty)^{2} \rightarrow(0,+\infty)$ is continuous;

$\left(C_{2}\right) a: \mathbf{T} \rightarrow(0,+\infty)$ is left dense continuous (i.e., $\left.a \in C_{\mathbf{l d}}(\mathbf{T},(0,+\infty))\right)$ and does not vanish identically on any closed subinterval of $[0, T]_{\mathbf{T}}$, where $C_{\mathbf{l d}}(\mathbf{T},(0,+\infty))$ denotes the set of all left dense continuous functions from $\mathbf{T}$ to $(0,+\infty)$;

$\left(C_{3}\right) \varphi:[-r, 0]_{\mathrm{T}} \rightarrow[0,+\infty)$ is continuous and $r>0$;

$\left(\mathrm{C}_{4}\right) \mu:[0, T]_{\mathrm{T}} \rightarrow[-r, T]_{\mathrm{T}}$ is continuous, $\mu(t) \leq t$ for all $t$;

$\left(C_{5}\right) B_{0}(v)$ is a continuous function defined on $\mathbb{R}$ and satisfies that there exist $B \geq 1$ and $A \geq 1$ such that

$$
B x \leq B_{0}(x) \leq A x \text { for all } \mathbb{R}
$$

Remark 1.1 Although the Banach space in this paper is the same as that of [8], i.e., $E=C_{\mathbf{l d}}^{\Delta}\left([0, T]_{\mathrm{T}}, \mathbb{R}\right)$ with $\|u\|=\max \left\{\max _{t \in[0, T]_{\mathbf{T}}}|u(t)|, \max _{t \in[0, T]_{\mathrm{T}^{k}}}\left|u^{\Delta}(t)\right|\right\}$, the hypotheses utilized in the existence theorem in this paper differ from those of [8] where the effect of $a(t)$ was imposed.

Throughout this work, we assume the knowledge of time scales and time-scale notation, first introduced by Hilger [12]. For more on time scales, please see the texts by Bohner and Peterson $[13,14]$.

In the remainder of this section, we state the following theorem which is crucial to our proof.

Let $\gamma$ and $\theta$ be nonnegative continuous convex functions on $P, \alpha$ be a nonnegative continuous concave function on $P$, and $\psi$ be a nonnegative continuous function on $P$. Then, for positive real numbers $a, b, c$ and $d$, we define the following convex sets:

$$
\begin{aligned}
& P(\gamma, d)=\{x \in P: \gamma(x)<d\}, \\
& P(\gamma, \alpha, b, d)=\{x \in P: b \leq \alpha(x), \gamma(x) \leq d\}, \\
& P(\gamma, \theta, \alpha, b, c, d)=\{x \in P: b \leq \alpha(x), \theta(x) \leq c, \gamma(x) \leq d\},
\end{aligned}
$$

and a closed set

$$
R(\gamma, \psi, a, d)=\{x \in P: a \leq \psi(x), \gamma(x) \leq d\} .
$$

To prove our main results, we need the following fixed point theorem due to Avery and Peterson in [11].

Theorem 1.1 Let $P$ be a cone in a real Banach space E. Let $\gamma$ and $\theta$ be nonnegative continuous convex functionals on $P, \alpha$ be a nonnegative continuous concave functional on $P$ and $\psi$ be a nonnegative continuous functional on P satisfying $\psi(\lambda x) \leq \lambda \psi(x)$ for $0 \leq \lambda \leq 1$, such that for some positive numbers $h$ and $d$,

$$
\alpha(x) \leq \psi(x) \quad \text { and } \quad\|x\| \leq h \gamma(x)
$$

for all $x \in \overline{P(\gamma, d)}$. Suppose that

$$
F: \overline{P(\gamma, d)} \rightarrow \overline{P(\gamma, d)}
$$


is completely continuous and there exist positive numbers $a, b$ and $c$ with $a<b$ such that:

(i) $\{x \in P(\gamma, \theta, \alpha, b, c, d): \alpha(x)>b\} \neq \phi$ and $\alpha(F x)>b$ for $x \in P(\gamma, \theta, \alpha, b, c, d)$;

(ii) $\alpha(F x)>b$ for $x \in P(\gamma, \alpha, b, d)$ with $\theta(F x)>c$;

(iii) $0 \notin R(\gamma, \psi, a, d)$ and $\psi(F x)<a$ for $x \in R(\gamma, \psi, a, d)$ with $\psi(x)=a$.

Then $F$ has at least three fixed points $x_{1}, x_{2}, x_{3} \in \overline{P(\gamma, d)}$ such that

$$
\begin{aligned}
& \gamma\left(x_{i}\right) \leq d \text { for } i=1,2,3, b<\alpha\left(x_{1}\right), \\
& a<\psi\left(x_{2}\right) \text { with } \alpha\left(x_{2}\right)<b \text { and } \psi\left(x_{3}\right)<a .
\end{aligned}
$$

\section{Main result}

In this section we consider the existence of three positive solutions for problem (1.1).

We say that $u$ is concave on $[0, T]_{\mathrm{T}}$ if $u^{\Delta \nabla}(t) \leq 0$ for $t \in[0, T]_{\mathrm{T}^{k} \cap \mathbf{T}_{k}}$.

We note that $u(t)$ is a solution of problem (1.1) if and only if

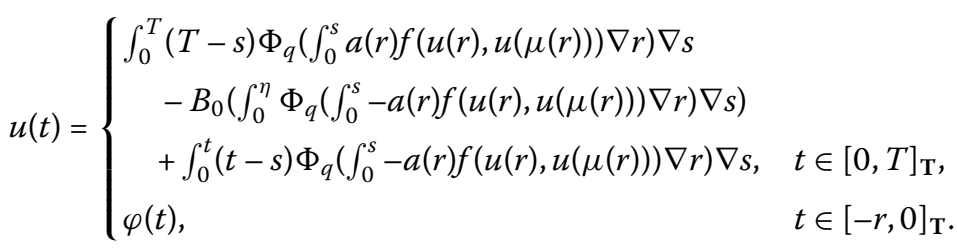

Let $E=C_{\mathbf{l d}}^{\Delta}\left([0, T]_{\mathrm{T}}, \mathbb{R}\right)$ with $\|u\|=\max \left\{\max _{t \in[0, T]_{\mathrm{T}}}|u(t)|, \max _{t \in[0, T]_{\mathrm{T}^{k}}}\left|u^{\Delta}(t)\right|\right\}, P=\{u \in$ $E: u$ is nonnegative, decreasing and concave on $\left.[0, T]_{\mathrm{T}}\right\}$. So $E$ is a Banach space with the norm $\|u\|$ and $P$ is a cone in $E$. For each $u \in E$, extend $u(t)$ to $[-r, T]_{\mathrm{T}}$ with $u(t)=\varphi(t)$ for $t \in[-r, 0]_{\mathrm{T}}$.

Define $F: P \rightarrow E$ by

$$
\begin{aligned}
(F u)(t)= & \int_{0}^{T}(T-s) \Phi_{q}\left(\int_{0}^{s} a(r) f(u(r), u(\mu(r))) \nabla r\right) \nabla s \\
& -B_{0}\left(\int_{0}^{\eta} \Phi_{q}\left(\int_{0}^{s}-a(r) f(u(r), u(\mu(r))) \nabla r\right) \nabla s\right) \\
& +\int_{0}^{t}(t-s) \Phi_{q}\left(\int_{0}^{s}-a(r) f(u(r), u(\mu(r))) \nabla r\right) \nabla s, \quad t \in[0, T]_{\mathrm{T}} .
\end{aligned}
$$

It is well known that this operator $F$ is completely continuous.

We seek a fixed point $u_{1}$ of $F$ in the cone $P$. Define

$$
u(t)= \begin{cases}\varphi(t), & t \in[-r, 0]_{\mathrm{T}}, \\ u_{1}(t), & t \in[0, T]_{\mathrm{T}} .\end{cases}
$$

Then $u(t)$ denotes a positive solution of problem (1.1).

Lemma 2.1 If $u \in P$, then

(i) $F u \in P$, i.e., $F: P \rightarrow P$.

(ii) $u(t) \geq \frac{T-t}{T} \max _{t \in[0, T]_{\mathbf{T}}}|u(t)|, t \in[0, T]_{\mathbf{T}}$.

(iii) $u(t)$ is decreasing on $t \in[0, T]_{\mathrm{T}}$. 
Proof This is easy, so we omit it here.

Let $l \in \mathbf{T}$ be fixed such that $0<l<\eta<T$, and set

$$
Y_{1}=\left\{t \in[0, T]_{\mathbf{T}}: \mu(t) \leq 0\right\} ; \quad Y_{2}=\left\{t \in[0, T]_{\mathbf{T}}: \mu(t)>0\right\} ; \quad Y_{3}=Y_{1} \cap[0, \eta]_{\mathbf{T}}
$$

Throughout this paper, we assume $Y_{3} \neq \phi$ and $\int_{Y_{3}} a(r) \nabla r>0$.

Define the nonnegative continuous concave functional $\alpha$, the nonnegative continuous convex functionals $\theta, \gamma$, and the nonnegative continuous functional $\psi$ on the cone $P$, respectively, as

$$
\begin{aligned}
& \gamma(u)=\|u\|, \quad \theta(u)=\max _{t \in[0, l]]_{\mathbf{T}^{k}}}\left|u^{\triangle}(t)\right|, \\
& \alpha(u)=\min _{t \in[l, \eta]_{\mathbf{T}}} u(t), \quad \psi(u)=\min _{t \in[0, \eta]_{\mathbf{T}}} u(t) .
\end{aligned}
$$

In addition, by Lemma 2.1, we have $\alpha(u)=\psi(u)=u(\eta)$ for each $u \in P$.

For convenience, we define

$$
\begin{aligned}
& \rho=T(B+2 T) \Phi_{q}\left(\int_{0}^{T} a(r) \nabla r\right), \quad \delta=A \int_{Y_{3}} \Phi_{q}\left(\int_{0}^{s} a(r) \nabla s\right) \nabla s, \\
& \lambda=T(B+T+\eta) \Phi_{q}\left(\int_{0}^{T} a(r) \nabla r\right) .
\end{aligned}
$$

We now state growth conditions on $f$ so that BVP (1.1) has at least three positive solutions.

Theorem 2.1 Let $0<\frac{T}{\eta} a<b<d, \rho b<\delta d$, and suppose that $f$ satisfies the following conditions:

$\left(\mathrm{H}_{1}\right) f(u, \varphi(s)) \leq \Phi_{p}\left(\frac{d}{\rho}\right)$ if $0 \leq u \leq d$ uniformly in $s \in[-r, 0]_{\mathrm{T}} ; f\left(u_{1}, u_{2}\right) \leq \Phi_{p}\left(\frac{d}{\rho}\right)$ if $0 \leq u_{i} \leq$ $d, i=1,2$,

$\left(\mathrm{H}_{2}\right) f(u, \varphi(s))>\Phi_{p}\left(\frac{b}{\delta}\right)$ if $b \leq u \leq d$ uniformly in $s \in[-r, 0]_{\mathrm{T}}$,

$\left(\mathrm{H}_{3}\right) f(u, \varphi(s))<\Phi_{p}\left(\frac{a}{\lambda}\right)$ if $0 \leq u \leq \frac{T}{\eta}$ a uniformly in $s \in[-r, 0]_{\mathbf{T}} ; f\left(u_{1}, u_{2}\right)<\Phi_{p}\left(\frac{a}{\lambda}\right)$ if $0 \leq u_{i} \leq$ $\frac{T}{\eta} a, i=1,2$.

Then BVP (1.1) has at least three positive solutions of the form

$$
u(t)= \begin{cases}u_{i}(t), & t \in[0, T]_{\mathrm{T}}, i=1,2,3 \\ \varphi(t), & t \in[-r, 0]_{\mathrm{T}}\end{cases}
$$

where $\gamma\left(u_{i}\right) \leq d$ for $i=1,2,3, b<\alpha\left(u_{1}\right), a<\psi\left(u_{2}\right)$ with $\alpha\left(u_{2}\right)<b$ and $\psi\left(u_{3}\right)<a$.

Proof We first assert that $F: \overline{P(\gamma, d)} \rightarrow \overline{P(\gamma, d)}$.

Let $u \in \overline{P(\gamma, d)}$, then $\gamma(u)=\|u\| \leq d$, consequently, $0 \leq u(t) \leq d$ for $t \in[0, T]_{\mathrm{T}}$. 
From $\left(\mathrm{H}_{1}\right)$, we have

$$
\begin{aligned}
& |F u(t)|=\int_{0}^{T}(T-s) \Phi_{q}\left(\int_{0}^{s} a(r) f(u(r), u(\mu(r))) \nabla r\right) \nabla s \\
& -B_{0}\left(\int_{0}^{\eta} \Phi_{q}\left(\int_{0}^{s}-a(r) f(u(r), u(\mu(r))) \nabla r\right) \nabla s\right) \\
& +\int_{0}^{t}(t-s) \Phi_{q}\left(\int_{0}^{s}-a(r) f(u(r), u(\mu(r))) \nabla r\right) \nabla s \\
& <\int_{0}^{T} T \Phi_{q}\left(\int_{0}^{T} a(r) f(u(r), u(\mu(r))) \nabla r\right) \nabla s \\
& +B \int_{0}^{T} \Phi_{q}\left(\int_{0}^{T} a(r) f(u(r), u(\mu(r))) \nabla r\right) \nabla s \\
& +\int_{0}^{T} T \Phi_{q}\left(\int_{0}^{T} a(r) f(u(r), u(\mu(r))) \nabla r\right) \nabla s \\
& =T(B+2 T) \Phi_{q}\left(\int_{Y_{1}} a(r) f(u(r), \varphi(\mu(r))) \nabla r+\int_{Y_{2}} a(r) f(u(r), u(\mu(r))) \nabla r\right) \\
& \leq T(B+2 T) \Phi_{q}\left(\int_{0}^{T} a(r) \nabla r\right) \frac{d}{\rho} \\
& =d \text {, } \\
& \left|(F u)^{\triangle}(t)\right|=\left|\int_{0}^{t} \Phi_{q}\left(\int_{0}^{s} a(r) f(u(r), u(\mu(r))) \nabla r\right) \nabla s\right| \\
& \leq \int_{0}^{T} \Phi_{q}\left(\int_{0}^{T} a(r) f(u(r), u(\mu(r))) \nabla r\right) \nabla s \\
& =T \Phi_{q}\left(\int_{Y_{1}} a(r) f(u(r), \varphi(\mu(r))) \nabla r+\int_{Y_{2}} a(r) f(u(r), u(\mu(r))) \nabla r\right) \\
& \leq T \Phi_{q}\left(\int_{0}^{T} a(r) \nabla r\right) \frac{d}{\rho} \\
& =\frac{d}{B+2 T} \\
& \leq d \text {. }
\end{aligned}
$$

Therefore $F(u) \in \overline{P(\gamma, d)}$, i.e., $F: \overline{P(\gamma, d)} \rightarrow \overline{P(\gamma, d)}$.

Secondly, we assert that $\{u \in P(\gamma, \theta, \alpha, b, c, d): \alpha(u)>b\} \neq \phi$ and $\alpha(F u)>b$ for $u \in$ $P(\gamma, \theta, \alpha, b, c, d)$.

Let $u(t)=k b$ with $k=\frac{\rho}{\delta}>1$, then $u(t)=k b>b$ and $\theta(u)=0<b$. Furthermore, by $\rho b<\delta d$ we have $\gamma(u) \leq d$. Let $c=k b$, then $\{u \in P(\gamma, \theta, \alpha, b, c, d): \alpha(u)>b\} \neq \phi$.

Moreover, $\forall u \in P(\gamma, \theta, \alpha, b, k b, d)$, we have $b \leq u(t) \leq d, t \in[0, \eta]_{\mathrm{T}}$.

From $\left(\mathrm{H}_{2}\right)$, we see that

$$
\begin{aligned}
\alpha(F u) & =(F u)(\eta) \\
& =\int_{0}^{T}(T-s) \Phi_{q}\left(\int_{0}^{s} a(r) f(u(r), u(\mu(r))) \nabla r\right) \nabla s
\end{aligned}
$$




$$
\begin{aligned}
& -B_{0}\left(\int_{0}^{\eta} \Phi_{q}\left(\int_{0}^{s}-a(r) f(u(r), u(\mu(r))) \nabla r\right) \nabla s\right) \\
& +\int_{0}^{\eta}(t-s) \Phi_{q}\left(\int_{0}^{s}-a(r) f(u(r), u(\mu(r))) \nabla r\right) \nabla s \\
\geq & -B_{0}\left(\int_{0}^{\eta} \Phi_{q}\left(\int_{0}^{s}-a(r) f(u(r), u(\mu(r))) \nabla r\right) \nabla s\right) \\
\geq & A\left(\int_{0}^{\eta} \Phi_{q}\left(\int_{0}^{s} a(r) f(u(r), u(\mu(r))) \nabla r\right) \nabla s\right) \\
\geq & A\left(\int_{Y_{3}} \Phi_{q}\left(\int_{0}^{s} a(r) f(u(r), \varphi(\mu(r))) \nabla r\right) \nabla s\right) \\
> & A \int_{Y_{3}} \Phi_{q}\left(\int_{0}^{s} a(r) \nabla s\right) \nabla s \frac{b}{\delta} \\
= & b,
\end{aligned}
$$

as required.

Thirdly, we assert that $\alpha(F u)>b$ for $u \in P(\gamma, \alpha, b, d)$ with $\theta(F u)>c$.

$\forall u \in P(\gamma, \alpha, b, d)$ with $\theta(F u)>k b$, from Lemma 2.1 we have

$$
\begin{aligned}
\theta(F u) & =\left|(F u)^{\triangle}(l)\right| \\
& =\int_{0}^{l} \Phi_{q}\left(\int_{0}^{s} a(r) f(u(r), u(\mu(r))) \nabla r\right) \nabla s>k b .
\end{aligned}
$$

So,

$$
\begin{aligned}
\alpha(F u)= & (F u)(\eta) \\
= & \int_{0}^{T}(T-s) \Phi_{q}\left(\int_{0}^{s} a(r) f(u(r), u(\mu(r))) \nabla r\right) \nabla s \\
& -B_{0}\left(\int_{0}^{\eta} \Phi_{q}\left(\int_{0}^{s}-a(r) f(u(r), u(\mu(r))) \nabla r\right) \nabla s\right) \\
& +\int_{0}^{\eta}(t-s) \Phi_{q}\left(\int_{0}^{s}-a(r) f(u(r), u(\mu(r))) \nabla r\right) \nabla s \\
\geq & -B_{0}\left(\int_{0}^{\eta} \Phi_{q}\left(\int_{0}^{s}-a(r) f(u(r), u(\mu(r))) \nabla r\right) \nabla s\right) \\
\geq & A\left(\int_{0}^{\eta} \Phi_{q}\left(\int_{0}^{s} a(r) f(u(r), u(\mu(r))) \nabla r\right) \nabla s\right) \\
\geq & A\left(\int_{0}^{l} \Phi_{q}\left(\int_{0}^{s} a(r) f(u(r), u(\mu(r))) \nabla r\right) \nabla s\right) \\
> & A k b=A \frac{\rho}{\delta} b \geq(A+T) b>b .
\end{aligned}
$$

This implies that $\alpha(F u)>b$ for $u \in P(\gamma, \alpha, b, d)$ with $\theta(F u)>c$.

Finally, we assert that $0 \notin R(\gamma, \psi, a, d)$ and $\psi(F u)<a$ for $u \in R(\gamma, \psi, a, d)$ with $\psi(u)=a$. As $\psi(0)=0<a$, we have $0 \notin R(\gamma, \psi, a, d) . \forall u \in R(\gamma, \psi, a, d)$ with $\psi(u)=\min _{t \in[0, \eta]_{\mathrm{T}}} u(t)=$ $u(\eta)=a$, by Lemma 2.1 we have $0 \leq u(t) \leq \frac{T}{T-\eta} a$ for $t \in[0, T]_{\mathrm{T}}$. 
From $\left(\mathrm{H}_{3}\right)$, we have

$$
\begin{aligned}
\psi(F u)= & (F u)(\eta) \\
= & \int_{0}^{T}(T-s) \Phi_{q}\left(\int_{0}^{s} a(r) f(u(r), u(\mu(r))) \nabla r\right) \nabla s \\
& -B_{0}\left(\int_{0}^{\eta} \Phi_{q}\left(\int_{0}^{s}-a(r) f(u(r), u(\mu(r))) \nabla r\right) \nabla s\right) \\
& +\int_{0}^{\eta}(t-s) \Phi_{q}\left(\int_{0}^{s}-a(r) f(u(r), u(\mu(r))) \nabla r\right) \nabla s \\
< & \int_{0}^{T} T \Phi_{q}\left(\int_{0}^{T} a(r) f(u(r), u(\mu(r))) \nabla r\right) \nabla s \\
& +B \int_{0}^{T} \Phi_{q}\left(\int_{0}^{T} a(r) f(u(r), u(\mu(r))) \nabla r\right) \nabla s \\
& +\int_{0}^{T} \eta \Phi_{q}\left(\int_{0}^{T} a(r) f(u(r), u(\mu(r))) \nabla r\right) \nabla s \\
= & T(B+T+\eta) \Phi_{q}\left(\int_{Y_{1}}^{T} a(r) f(u(r), \varphi(\mu(r))) \nabla r+\int_{Y_{2}} a(r) f(u(r), u(\mu(r))) \nabla r\right) \\
\leq & T(B+T+\eta) \Phi_{q}\left(\int_{0}^{T} a(r) \nabla r\right) \frac{a}{\lambda} \\
= & a,
\end{aligned}
$$

which shows that condition (iii) of Theorem 1.1 is fulfilled.

Thus, all the conditions of Theorem 1.1 are satisfied. Hence, $F$ has at least three fixed points $u_{1}, u_{2}, u_{3}$ satisfying

$$
\gamma\left(u_{i}\right) \leq d \text { for } i=1,2,3, b<\alpha\left(u_{1}\right), a<\psi\left(u_{2}\right) \text { with } \alpha\left(u_{2}\right)<b \text { and } \psi\left(u_{3}\right)<a \text {. }
$$

Let

$$
u(t)= \begin{cases}u_{i}(t), & t \in[0, T]_{\mathrm{T}}, i=1,2,3 \\ \varphi(t), & t \in[-r, 0]_{\mathrm{T}}\end{cases}
$$

which are three positive solutions of BVP (1.1)

\section{Competing interests}

The author declares that she has no competing interests.

\section{Acknowledgements}

The author thanks the referees and the editors for their helpful comments and suggestions. Research was supported by the Natural Science Foundation of Gansu Province of China (Grant no. 1310RJYA080).

Received: 17 June 2014 Accepted: 14 August 2014 Published: 24 Sep 2014

\section{References}

1. Guan, W: Three positive solutions for $p$-Laplacian functional dynamic equations on time scales. Electron. J. Qual. Theory Differ. Equ. 28, 1-7 (2008). http://www.math.u-szeged.hu/ejqtde/

2. Kaufmann, ER, Raffoul, YN: Positive solutions for a nonlinear functional dynamic equation on a time scale. Nonlinear Anal. 62, 1267-1276 (2005) 
3. Song, CX, Xiao, CT: Positive solutions for $p$-Laplacian functional dynamic equations on time scales. Nonlinear Anal. 66 , 1989-1998 (2007)

4. Song, CX, Weng, PX: Multiple positive solutions for $p$-Laplacian functional dynamic equations on time scales. Nonlinear Anal. 68, 208-215 (2008)

5. Song, CX, Gao, XJ: Positive solutions for third-order $p$-Laplacian functional dynamic equations on time scales. Bound. Value Probl. 2011, 585378 (2011)

6. Wang, DB: Three positive solutions for $p$-Laplacian functional dynamic equations on time scales. Electron. J. Differ. Equ. 2007(95), 1-9 (2007)

7. Wang, DB, Guan, W: Multiple positive solutions for $p$-Laplacian functional dynamic equations on time scales. Taiwan. J. Math. 12, 2327-2340 (2008)

8. Wang, DB, Guan, W: Multiple positive solutions for third-order $p$-Laplacian functional dynamic equations on time scales. Adv. Differ. Equ. (2014). doi:10.1186/1687-1847-2014-145

9. Avery, Rl, Chyan, CJ, Henderson, J: Twin solutions of boundary value problems for ordinary differential equations and finite difference equations. Comput. Math. Appl. 42, 695-704 (2001)

10. Leggett, RW, Williams, LR: Multiple positive fixed points of nonlinear operators on ordered Banach spaces. Indiana Univ. Math. J. 28, 673-688 (1979)

11. Avery, Rl, Peterson, AC: Three positive fixed points of nonlinear operators on ordered Banach spaces. Comput. Math. Appl. 42, 313-322 (2001)

12. Hilger, S: Analysis on measure chains - a unified approach to continuous and discrete calculus. Results Math. 18, 18-56 (1990)

13. Bohner, M, Peterson, A: Dynamic Equations on Time Scales: An Introduction with Applications. Birkhäuser, Boston (2001)

14. Bohner, M, Peterson, A: Advances in Dynamic Equations on Time Scales. Birkhäuser, Boston (2003)

10.1186/1687-1847-2014-242

Cite this article as: Guan: Existence of multiple positive solutions for third-order $p$-Laplacian functional dynamic equations on time scales. Advances in Difference Equations 2014, 2014:242

\section{Submit your manuscript to a SpringerOpen ${ }^{\odot}$ journal and benefit from:}

- Convenient online submission

- Rigorous peer review

- Immediate publication on acceptance

- Open access: articles freely available online

- High visibility within the field

- Retaining the copyright to your article 